\title{
Calibração do Storm Water Management Model (SWMM) utilizando algoritmos evolucionários multiobjetivo
}

\author{
Storm Water Management Model \\ calibration using multiobjective evolutionary algorithms
}

Klebber Teodomiro Martins Formiga', Maira de Carvalho², Karla Alcione Silva ${ }^{3}$, Alexandre Kepler Soares ${ }^{1}$

\begin{abstract}
RESUMO
O estudo teve por objetivo a realização da calibração do modelo hidrológico Storm Water Management Model (SWMM) para a Bacia Hidrográfica do Arroio Cancela, localizada em Santa Maria, Rio Grande do Sul, utilizando o algoritmo evolucionário multiobjetivo R-NSGA. Para tanto, foram realizadas modificações na estrutura do SWMM, de modo que permitisse seu acoplamento como Evolucionary Reference Point Based Non-Dominated Sorting Genetic Algorithm (R-NSGA) em ambiente de programação MATLAB. As funções objetivo utilizadas foram o Coeficiente de Eficiência de Nash-Sutcliffe (COE), o Erro da Vazão de Pico (EQP) e o Erro do Volume Escoado (EVOL) aplicadas simultaneamente na calibração do modelo. Foi proposto um método para determinação da maior compatibilidade de modo a elencar as melhores soluções. Os resultados dos parâmetros calibrados do SWMM foram próximos aos valores físicos da bacia, com exceção dos valores relativos à equação de Horton. As soluções de maior compatibilidade apresentam um melhor comportamento para os eventos de validação, evidenciando a importância da otimização multiobjetivo.
\end{abstract}

Palavras-chave: otimização multiobjetivo; vazão de pico; modelos hidrológicos.

\begin{abstract}
This paper focused on the calibration of Storm Water Management Model (SWMM) for the Cancela River Basin, located in Santa Maria, Rio Grande do Sul, Brazil, using the multi-objective Evolucionary Reference Point Based Non-Dominated Sorting Genetic Algorithm R-NSGA. Modifications were made in SWMM structure to allow its linking with R-NSGA in MATLAB. The objective functions proposed were the efficiency coefficient of Nash-Sutcliffe (eNS), the Peak Flow Error (EQ) and Runoff Volume error (EVOL), which were applied simultaneously to the model calibration. A method for determining the maximum compatibility to rank the best solutions is proposed. The parameters estimated by SWMM calibration were close to physical values of the basin, except those relating to the Horton equation in the most compatible solutions have a better behavior for the validation event, highlighting the importance of multi-objective optimization.
\end{abstract}

Keywords: multiobjective optimization; peak flow; hydrologic models.

\section{INTRODUÇÃO}

Parte considerável das bacias hidrográficas brasileiras que não está localizada na região amazônica sofre ou sofreu grandes influências de atividades antrópicas nas últimas décadas. Como consequências dessas alterações, vieram acompanhadas mudanças no comportamento do regime de vazões, em especial, nas vazões de pico. A alteração do terreno natural, substituído muitas vezes por pastagens, cultivos e pela urbanização, provoca um aumento significativo das vazões extremas, gerando inúmeras consequências sociais, econômicas e ambientais. A morfologia dos cursos d'água não está preparada para comportar esse novo patamar de vazões, ocasionando processos erosivos, em especial, nas pequenas bacias de cabeceira (DAMÉ et al., 2010).

Para se evitar o aumento da degradação desses meios, têm-se buscado formas de reduzir o volume escoado e as vazões de cheias. Com esse intuito,

'Doutor em Hidraulica e Saneamento pela Universidade de São Paulo (USP) - São Paulo (SP), Brasil.

${ }^{2}$ Mestre em Engenhaira do Meio Ambiente pela Universidade Federal de Goiás (UFG) - Goiânia (GO), Brasil.

${ }^{3}$ Doutora em Ciencias Ambientais pela UFG - Goiânia (GO), Brasil.

Endereço para correspondência: Klebber Teodomiro Martins Formiga - Avenida Universitária, 1488, bloco A, sala 8 - CEP: 74605-220 - Goiânia (GO), Brasil -

E-mail: klebber.formiga@gmail.com

Recebido: 12/03/14 - Aceito: 01/03/16 - Reg. ABES: 131862 
tem-se recorrido a medidas mitigadoras, que, no caso do meio urbano, envolvem a construção de reservatórios de detenção e o uso de medidas de controle na fonte, enquanto, no meio rural, técnicas de plantio direto e pequenos reservatórios são mais comuns (NASCIMENTO et al., 2007; KIM et al., 2014; BARROS et al., 2011). Para implementação das medidas citadas é necessário, primeiramente, avaliar o efeito das possíveis modificações a serem realizadas e as soluções mais eficientes para cada situação, empregando na maioria dos casos modelos hidrológicos/hidráulicos para as condições atuais e futuras da bacia (LIONG \& YBRAHIM, 1994).

O desenvolvimento de um modelo hidrológico envolve etapas como:

1. identificação das características superficiais da bacia, principalmente em termos de áreas impermeabilizadas, inclinação do terreno e uso do solo;

2. determinação das dimensões e características morfológicas da bacia; e

3. definição dos parâmetros hidráulicos e hidrológicos dos diversos componentes do modelo.

Nas duas primeiras etapas, normalmente, utilizam-se procedimentos baseados em Sistemas de Informações Geográficas (SIG) (LIONG; CHAN; LUM, 1991). Já na última fase, devido à complexidade do sistema estudado, e em virtude das inevitáveis simplificações de modelos matemáticos que os descrevem, alguns parâmetros devem ser determinados experimentalmente através de observações de campo, enquanto outros podem ser estimados com base nas características da bacia (MUKOLWE et al., 2014).

Para a estimativa dos parâmetros do modelo, é necessário realizar procedimentos de calibração, ou seja, utilizar um método eficiente de determinação do conjunto de soluções que minimizem e/ou maximizem alguns critérios que expressam o grau de concordância entre os resultados do modelo simulado e os dados medidos em campo (GUPTA \& SOROOSHIAN, 1985).

De um modo geral, no processo de calibração são encontradas dificuldades relacionadas, principalmente, em se ajustar os parâmetros para representar as diferentes condições do sistema. Essas condições são geradas pelos múltiplos eventos que podem ser observados, bem como pela distribuição de diferentes pontos de medição ao longo da bacia. Seibert e McDonnell (2002) e Reed et al. (2013) mostram que, nesta situação, os métodos de otimização mono-objetivo (de único objetivo) enfrentam dificuldades em encontrar um conjunto de parâmetros confiável para o problema. Uma das formas sugeridas para enfrentar esse tipo de dificuldade é empregar métodos multiobjetivo, em que se podem utilizar vários critérios de avaliação do modelo (KREBS et al., 2013).

Dentro do contexto multiobjetivo, várias funções objetivo (FO) podem ser consideradas simultaneamente no processo, de modo a se considerar os diferentes eventos avaliados sem, no entanto, perder informações. Uma das dificuldades enfrentadas ao se lidar com estes métodos reside no fato de se ter várias soluções como resposta. No entanto, tal empecilho pode ser superado empregando-se técnicas específicas para definição do conjunto de parâmetros a ser escolhido. Dessa maneira, os algoritmos evolucionários (AE) tornaram-se a principal ferramenta para a análise de problemas multiobjetivo, principalmente os relacionados aos recursos hídricos (REED et al., 2013). A premissa é que, em se trabalhar com um conjunto de soluções simultâneas, e não com apenas com um único valor ótimo, possibilita-se o uso dos AE como uma plataforma ideal para a otimização multiobjetivo, principalmente para a calibração de parâmetros de modelos hidrológicos (TANG; REED; WAGENER, 2006).

Existem diversos modelos hidrológicos para várias finalidades. Os modelos hidrológicos disponíveis com o objetivo de determinar vazões de cheia em bacias hidrográficas são denominados de chuva-vazão e, como exemplos, têm-se: o MOdel for Urban Sewers (MOUSE) (DHI, 1995), o Hydrological Model System (HEC-HMS) (SCHARFFENBERG, 2015) e o Storm Water Management Model (SWMM) (ROSSMAN, 2010).

O SWMM foi desenvolvido pela United States Environmental Protection Agency (EPA) para a simulação quali-quantitativa do escoamento de pequenas bacias, sendo capaz de simular aspectos dos ciclos hidrológicos e da qualidade da água. Durante as últimas décadas, o SWMM tem sido foco de muitos estudos que abordam a calibração empregando otimização mono-objetivo (LIONG \& IBRAHIM, 1994, BARCO; WONG; STENSTROM, 2008; KIM et al., 2014) e multiobjetivo (KREBS et al., 2013; MULETA et al., 2013).

Dessa forma, este estudo visou realizar a calibração multiobjetivo do SWMM para a Bacia Hidrográfica do Arroio Cancela, localizada em Santa Maria, Rio Grande do Sul, pela utilização do Evolucionary Reference Point Based Non-Dominated Sorting Genetic Algorithm (R-NSGA-II) proposto por Deb et al. (2006).

\section{MATERIAIS E MÉTODOS}

\section{Área de estudo}

A Bacia Hidrográfica do Arroio Cancela está localizada na área do município de Santa Maria, no Estado do Rio Grande do Sul. Possui área de contribuição de $4,95 \mathrm{~km}^{2}$, comprimento do rio principal de $1.343 \mathrm{~m}$ e exutório nas coordenadas 227549.66E e 6710158.57S, conforme Figura 1.

\section{Storm Water Management Model}

O SWMM foi escolhido por apresentar grande aceitação por parte da comunidade científica e por sua capacidade em simular o escoamento a partir de chuvas em pequenas bacias rurais e urbanas (BARCO; WONG; STENSTROM, 2008).

Com o intuito de testar a capacidade do método de otimização foi proposta uma configuração simplificada da bacia, em que ela é considerada como concentrada, sendo representada por apenas uma sub-bacia no SWMM. Esse tipo de representação, embora possa ser considerada muito simples, apresentou resultados satisfatórios em problemas em 
que o nível de incerteza dos parâmetros era elevado, obtendo resultados próximos dos encontrados quando se tem um detalhamento maior do ambiente (SEIBT, 2013).

A sub-bacia no SWMM é composta de duas partes, uma permeável e outra impermeável, sendo a porcentagem da área impermeável um dos parâmetros do modelo. O modelo empregado para separação de escoamento foi o método de Horton. Para a propagação da cheia nos planos das sub-bacias, foi utilizado o modelo de Onda Cinemática, onde a rugosidade da superfície e a inclinação são os parâmetros que interferem no fenômeno (ROSSMAN, 2010).

As variáveis hidrológicas foram obtidas por meio de monitoramentos pluviométrico e fluviométrico realizados para a bacia por Garcia e Paiva (2005), conforme estações dispostas na Figura 1.

Ao todo, foram considerados três eventos isolados de alta intensidade tidos como os mais representativos para o estudo, a fim de se avaliar a capacidade do modelo em gerar resultados próximos da realidade (Figura 2).

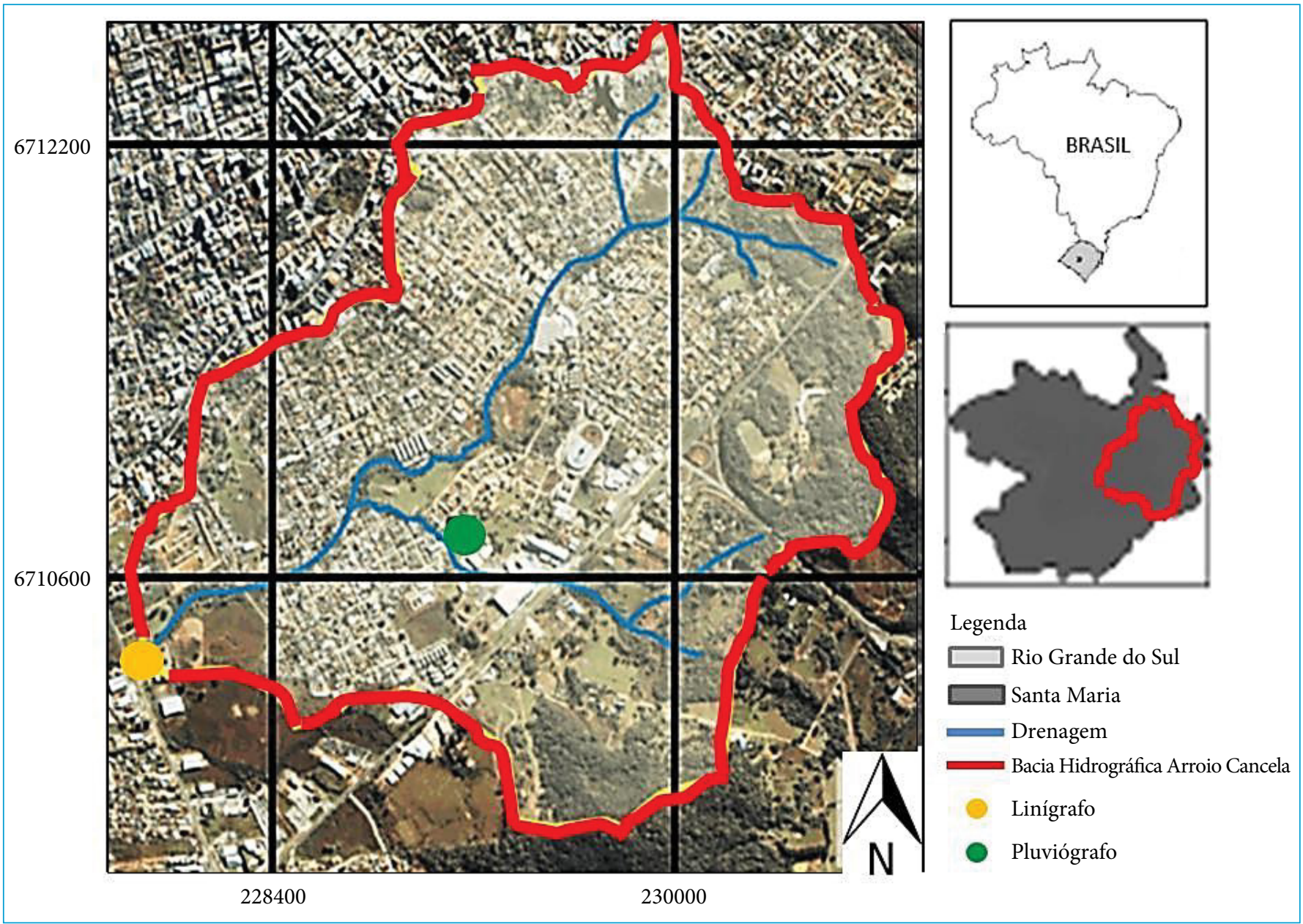

Figura 1 - Localização da Bacia Hidrográfica do Arroio Cancela, Santa Maria (RS).

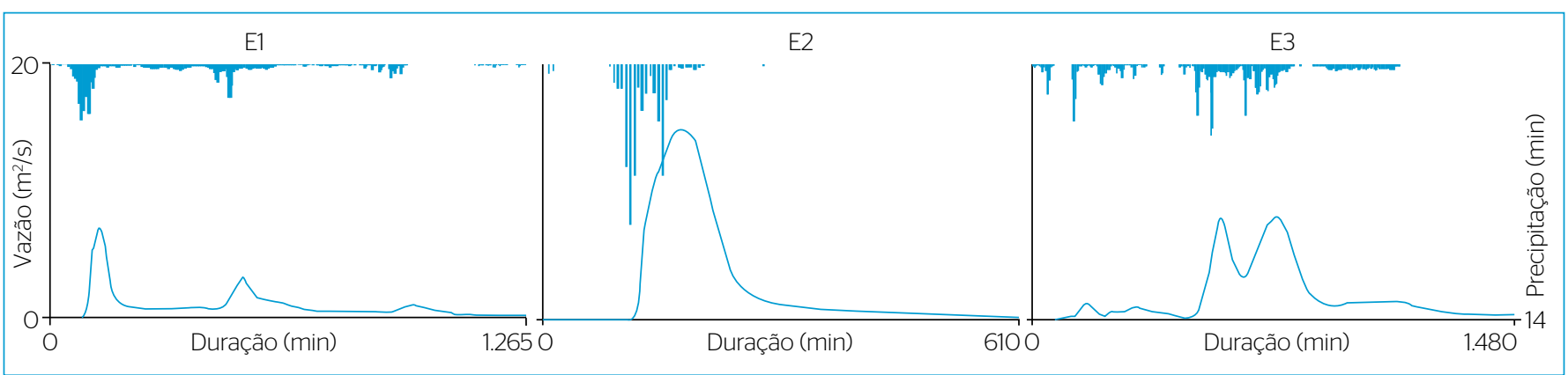

Figura 2 - Hidrogramas e hietogramas dos eventos 1, 2, e 3 (E1, E2 e E3) empregados na análise do Storm Water Management Model para a Bacia Hidrográfica do Arroio Cancela. 
Dessa forma, o evento 1 (E1) foi empregado para a calibração, enquanto os eventos 2 e 3 (E2 e E3) foram utilizados para a validação do modelo. Para análise do método, empregaram-se apenas eventos com forte escoamento uma vez que chuvas de pequena intensidade tendem a não serem bem representadas por modelos concentrados.

Foram considerados os seguintes parâmetros na calibração do SWMM: coeficiente de Manning para área impermeável $\left(\mathrm{n}_{\mathrm{i}}\right)$; coeficiente de Manning para a área permeável $\left(\mathrm{n}_{\mathrm{p}}\right)$; armazenamento em depressões para a área impermeável $\left(\mathrm{D}_{\mathrm{i}}\right)$; armazenamento em depressões para a área permeável $\left(\mathrm{D}_{\mathrm{p}}\right)$; porcentagem de área impermeável $\left(\mathrm{A}_{\mathrm{i}}\right)$; largura de escoamento $(\mathrm{W})$; declividade do plano (i); taxa inicial de infiltração $\left(f_{o}\right)$; capacidade limite de infiltração $\left(f_{c}\right)$ e taxa de decaimento (a). A faixa de variação dos parâmetros adotada na calibração é apresentada na Tabela 1.

\section{Interface Storm Water Management Model-MATLAB}

Foi realizada alteração na estrutura do código do SWMM a fim de permitir as modificações instantâneas nos parâmetros empregados na otimização e leitura dos resultados do modelo como ponteiros. Dessa maneira, trabalhou-se apenas em nível de memória RAM dentro do ambiente MATLAB, o que aumentou significativamente a velocidade de processamento e o processo

Tabela 1 - Parâmetros empregados na calibração com unidades e faixa de valores adotada.

\begin{tabular}{|c|c|c|c|c|}
\hline Parâmetro & Sigla & Unidade & $\begin{array}{l}\text { Limite } \\
\text { superior }\end{array}$ & $\begin{array}{l}\text { Limite } \\
\text { inferior }\end{array}$ \\
\hline Largura & W & $\mathrm{m}$ & 1 & 100 \\
\hline Declividade & i & $\%$ & 50 & 1000 \\
\hline Áreas impermeáveis & $A_{i}$ & $\%$ & 0.01 & 10 \\
\hline $\begin{array}{l}\text { Coeficiente de rugosidade } \\
\text { de Manning - Superfícies } \\
\text { impermeáveis }\end{array}$ & $n_{i}$ & - & 0.01 & 0.1 \\
\hline $\begin{array}{l}\text { Coeficiente de rugosidade } \\
\text { de Manning - superfícies } \\
\text { permeáveis }\end{array}$ & $n_{p}$ & - & 0.02 & 0.3 \\
\hline $\begin{array}{l}\text { Capacidade de } \\
\text { armazenamento em } \\
\text { depressões - superfícies } \\
\text { impermeáveis }\end{array}$ & $D_{i}$ & $\mathrm{~mm}$ & 1 & 100 \\
\hline $\begin{array}{l}\text { Capacidade de } \\
\text { armazenamento em } \\
\text { depressões - superfícies } \\
\text { permeáveis }\end{array}$ & $D_{p}$ & $\mathrm{~mm}$ & 1 & 100 \\
\hline Taxa inicial de infiltração & $f_{0}$ & $\mathrm{~mm} \cdot \mathrm{h}^{-1}$ & 1 & 50 \\
\hline $\begin{array}{l}\text { Capacidade limite de } \\
\text { infiltração }\end{array}$ & $f_{c}$ & $\mathrm{~mm} \cdot \mathrm{h}^{-1}$ & 1 & 100 \\
\hline $\begin{array}{l}\text { Taxa de decaimento da } \\
\text { infiltração }\end{array}$ & a & $h^{-1}$ & 0.1 & 10 \\
\hline
\end{tabular}

de modelagem. Um fluxograma do funcionamento do método é apresentado na Figura 3.

O SWMM foi recompilado de modo a aceitar as entradas do otimizador no MATLAB e fornecer como resposta uma série de vazões para um evento. O SWMM foi inicialmente compilado, criando-se uma biblioteca SWMM5.dll e um arquivo SWMM5.h. Ao rodar o SWMM no MATLAB usando o arquivo dlle o comando calllib, três passos precisam ser feitos:

1. Abertura do modelo (swmm_open), que requer os dados de entrada contidos no arquivo inp.

2. A simulação propriamente dita utilizando a função principal swmm_run, em que são enviados os parâmetros calculados pelo algoritmo otimizador e enviada a resposta do modelo.

3. O encerramento do processo com a função swmm_close, em que é feita a desalocação das variáveis (Figura 3).

Assim, os comandos adotados no código do MATLAB foram: - callib('SWMM5,'swmm_open,'inpfile.inp','reportfile.rpt,',binfile.bin');

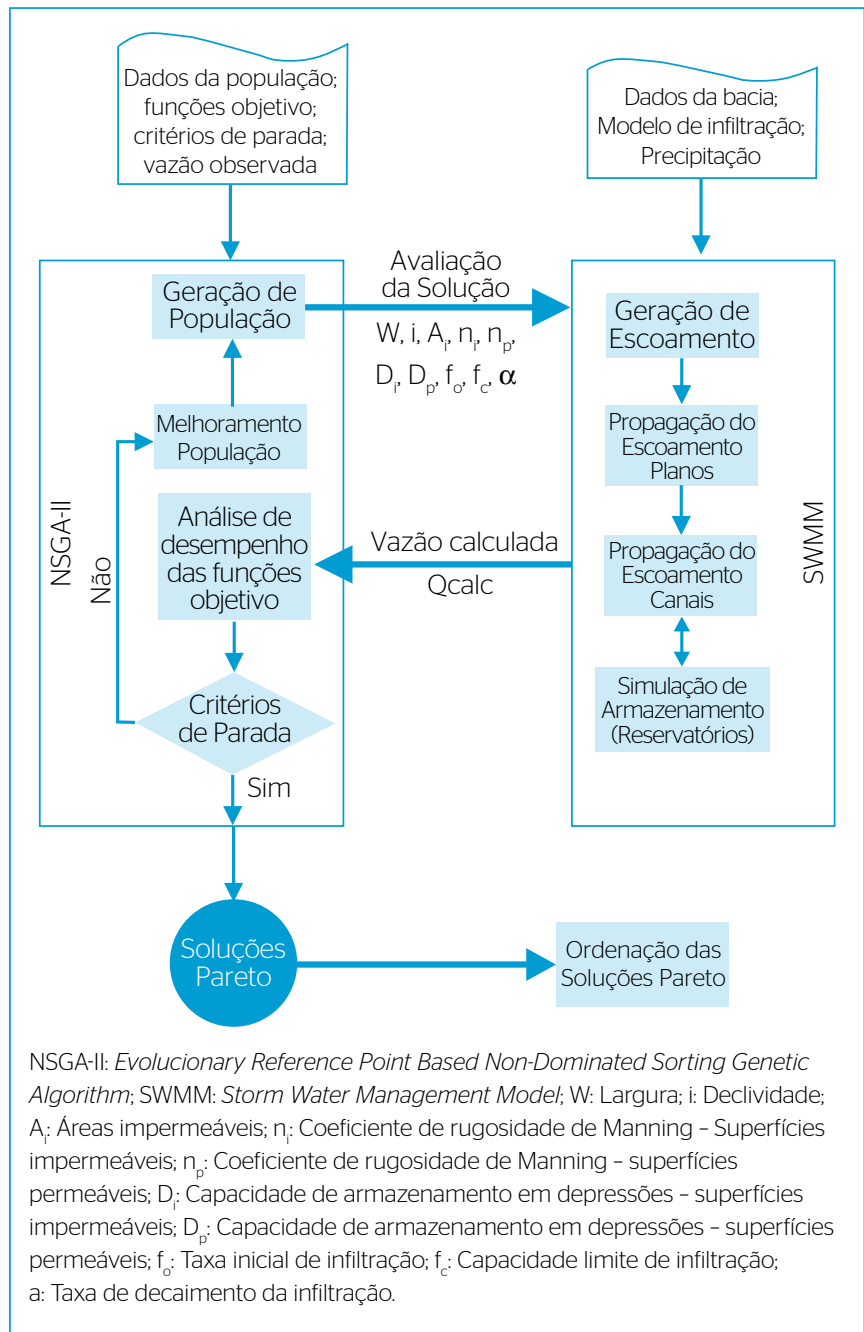

Figura 3 - Fluxograma do método de calibração. 
- callib('SWMM5',swmm_run',' inpfile.inp',entrada,saida);

- callib('SWMM5',swmm_close').

A interface interna no SWMM foi feita empregando a seguinte

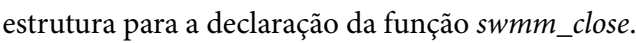

- swmm_run $\left(\right.$ char ${ }^{*} f 1$, struct c_struct ${ }^{*}$ entrada, double ${ }^{* *}$ sai).

O arquivo de entrada é uma estrutura e o de saída é um vetor, sendo todos eles tratados como ponteiros. Assim, com a variável entrada contendo todos os parâmetros que o NSGA-II envia para o SWMM, é preciso apenas fazer a alteração específica dentro do código alterando a variável desejada. Esse procedimento pode ser adaptado e empregado para qualquer variável que se deseja alterar em um processo externo ao SWMM dentro do MATLAB.

\section{Funções objetivo}

As diretrizes metodológicas adotadas para calibração do modelo foram adaptadas de Neufville, Schaake e Stafford (1971), em que a solução de um problema multiobjetivo deve ser dividida em cinco partes, a saber:

1. Escolha dos objetivos.

2. Definição das funções objetivo.

3. Geração de alternativas.

4. Avaliação das alternativas.

5. Escolha ou ordenação das soluções do problema (Formiga, 2005).

Dessa forma, na etapa de escolha dos objetivos, visa-se identificar os fatores que podem interferir na definição entre duas soluções. Assim, os objetivos escolhidos são: a proximidade do valor da vazão de pico calculada com a observada; forma similar dos hidrogramas simulados e observados; e concordância entre o volume escoado simulado e observado.

$\mathrm{Na}$ definição das funções objetivo, etapa em que os objetivos escolhidos são quantificados numericamente de modo que se possam comparar objetivamente duas alternativas, foram utilizadas três funções de avaliação para ajuste do modelo (Equações 1, 2 e 3). Essas funções foram propostas por ASCE Task committee (1993), Krause, Boyle e Bäse (2005) e Moriasi et al. (2007), e aferem o erro do ajuste do hidrograma: coeficiente de eficiência de Nash e Sutcliffe (1970) - (COE), o erro percentual do pico de vazão (EPQ) e o erro percentual do volume escoado (EVOL), respectivamente.

Máx.FO1 =1 $-\frac{\sum_{i-1}^{N t}\left(Q_{o b s}-Q_{c a l c}\right)^{2}}{\sum_{i=1}^{N t}\left(Q_{o b s}-\overline{Q_{o}}\right)^{2}}$

Mín.FO2 $=\left|\max _{o b s}-\max Q_{\text {calc }}\right| / \max _{o b s} \cdot 100$
Mín.FO3 $=\left|V o l_{o b s}-V o l_{c a l c}\right| / V o l_{o b s} .100$

Em que:

$Q_{o b s}$ é a vazão observada $\left(\mathrm{m}^{3} \cdot \mathrm{s}^{-1}\right)$;

$Q_{\text {calc }}$ é a vazão calculada $\left(\mathrm{m}^{3} \cdot \mathrm{s}^{-1}\right)$;

$Q_{o}$ é a média das vazões observadas $\left(\mathrm{m}^{3} \cdot \mathrm{s}^{-1}\right)$;

$\mathrm{N}_{\mathrm{t}}$ é o número de intervalos de tempo do evento analisado;

Vol $_{\text {obs }}$ é o volume observado $\left(\mathrm{m}^{3}\right)$;

e $\operatorname{Vol}_{\text {calc }}$ é o volume calculado $\left(\mathrm{m}^{3}\right)$.

\section{Evolucionary Reference Point Based Non- Dominated Sorting Genetic Algorithm - II}

$\mathrm{Na}$ etapa de geração de alternativas, procuram-se encontrar possíveis soluções para o problema, ou seja, encontrar conjuntos de alternativas Pareto. No caso de otimização multiobjetivo, essas soluções pertencem ao conjunto de soluções não dominadas, devendo ser encontradas por um método específico. Para tanto, empregou-se o método NSGA-II (Deb et al., 2000), que usa alguns conceitos do método NSGA (SRINIVAS \& DEB, 1994). Mesmo possuindo semelhanças com o NSGA, este apresenta uma abordagem metodológica diferente e emprega o conceito de elitismo. Assim, o NSGA-II foi escolhido para o estudo em razão de ser considerado como um dos melhores algoritmos de otimização multiobjetivo da atualidade (Reed et al., 2013). A versão empregada no estudo foi a R-NSGA-II, desenvolvida para o MATLAB por Lin (2011).

Foi realizada inicialmente análise de determinação dos parâmetros para o método de otimização R-NSGA-II, em que o evento E1 foi simulado com diversas combinações dos parâmetros do modelo de otimização até a sua definição. Foram testados o tamanho da população, o número de gerações, o tipo de função escala, a função de seleção, a função mutação, a taxa de mutação, a função de recombinação ou crossover e a taxa do crossover.

A avaliação das alternativas foi realizada por meio do modelo hidrológico, comparando os resultados obtidos com os observados em campo, empregando, para tanto, as FO. A escolha da solução do problema foi feita a partir da comparação das diferentes alternativas encontradas pelo algoritmo de geração da frente Pareto. Para isso, utilizou-se o método de melhor compatibilidade ou compromisso, em que os valores das FO das soluções Pareto-ótimas encontradas são normalizados com relação à média e ao desvio pelas Equações 4 e 5 :

$\left\|F O_{i}\right\|=\frac{F O_{i}-\overline{F O_{i}}}{\sigma_{F O_{i}}}$ para maximização do valor de $\mathrm{FO}$ ou

$\left\|F O_{i}\right\|=-\frac{F O_{i}-\overline{F O_{i}}}{\sigma_{F O_{i}}}$ para minimização do valor de $\mathrm{FO}$ 
Em que: $\left|F O_{i}\right| \mid$ o valor normalizado da função objetivo;

$\overline{F_{i}}$ a média dos valores das funções objetivo i do conjunto de soluções encontrado;

e $\sigma_{F O_{i}}$ o desvio padrão dos valores funções objetivo i do conjunto de soluções encontrado.

O índice de compatibilidade (C), que indica a melhor adequação da solução diante dos diferentes objetivos, foi calculado pela soma das funções normalizadas, em que quanto maior o seu valor, mais compatível é a alternativa.

\section{RESULTADOS E DISCUSSÃO}

\section{Calibração}

O NSGA-II foi executado 10 vezes para a calibração do modelo considerando o evento $\mathrm{E} 1$, em que os parâmetros obtidos para o algoritmo após simulação com diversas combinações dos critérios foram:

- Tamanho da População: 400;

- Número de Geração: 200;

- Função de Escala: ranking;

- Função de Seleção: torneio;

- Função de Mutação: uniforme;

- Taxa de Mutação: 0,02;
- Função de Recombinação: dois pontos;

- Taxa de Recombinação: 0,8.

O tempo computacional empregado para cada processo de otimização foi de 12 minutos, utilizando um computador Intel Core i7 930 RAM 8 GB. Após obtenção dos 10 grupos de soluções, foi extraído o conjunto de soluções não dominadas. Para o total de 4.000 soluções obtidas, foi realizada análise a fim de se encontrar as soluções não dominadas, obtendo-se um valor de 1.235 alternativas.

A Figura 4 apresenta a matriz gráfica com o resultado da distribuição dos parâmetros e as suas relações, com destaque para a análise de frequência de cada parâmetro na diagonal. A frente Pareto após o término do processo de escolha das soluções não dominadas é apresentada na Figura 5.

Verifica-se, de acordo com a Figura 4, que o valor da rugosidade de Manning para a área impermeável $\left(\mathrm{n}_{\mathrm{i}}\right)$ apresentou maior prevalência - em torno de 0,016, valor representativo para o asfalto/cimento, que é a superfície mais comum para essa situação. Para a área permeável, o valor mais frequente ficou em torno de 0,025 , que está associado a escoamento em superfícies mais rugosas, como o solo natural. O armazenamento em depressões mostrou uma variação maior, com valores que variam entre 3 e 7 mm para a área impermeável $\left(\mathrm{D}_{\mathrm{i}}\right)$, e entre 4 e $15 \mathrm{~mm}$ para a área permeável $\left(\mathrm{D}_{\mathrm{p}}\right)$. Essas faixas de valores para o coeficiente de Manning e para o armazenamento em depressões

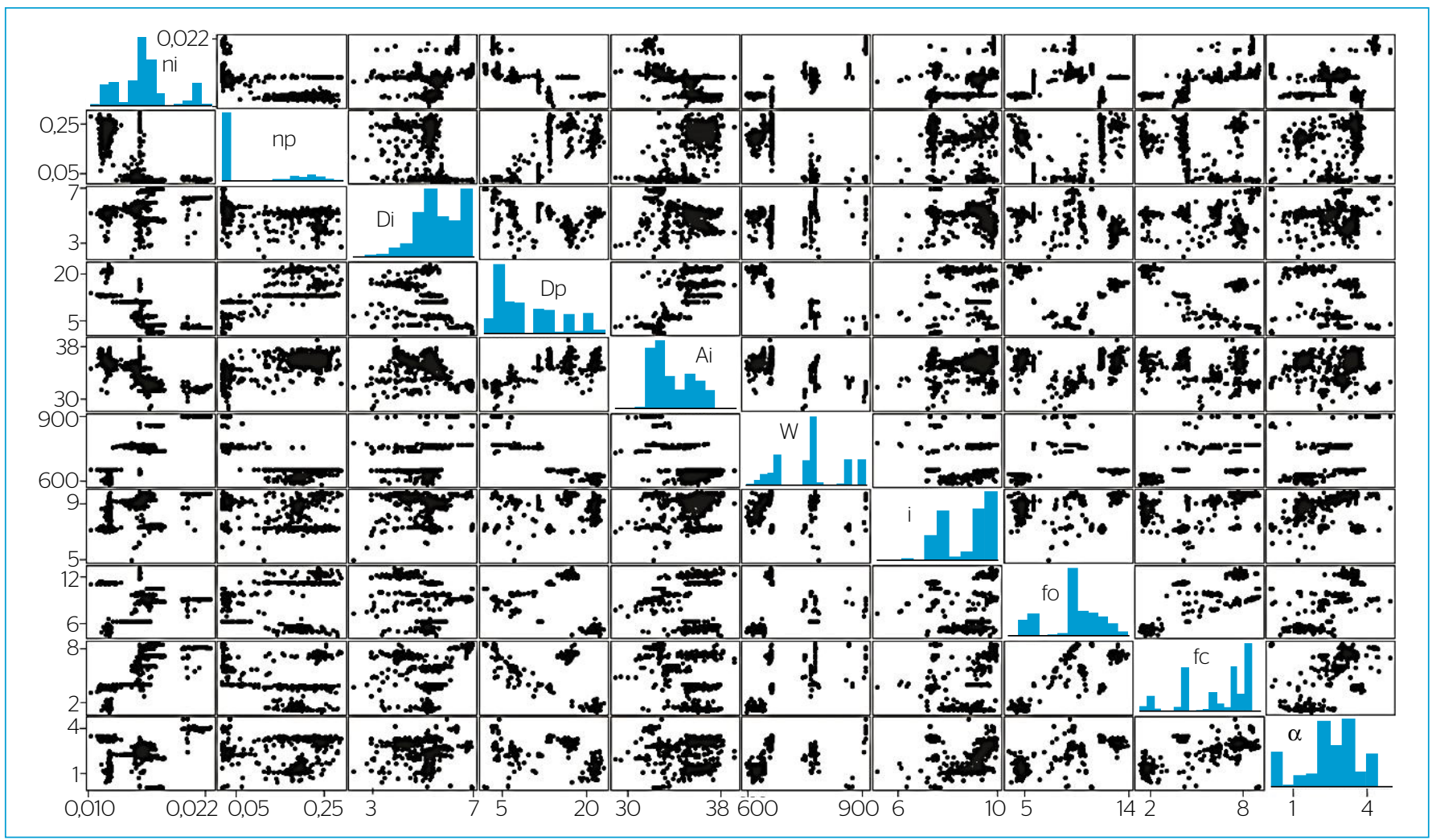

Figura 4 - Matriz dos gráficos da relação dos parâmetros do modelo e o histograma de sua distribuição (diagonal). 
encontram-se próximas às que constam na literatura para locais semelhantes (GARCIA \& PAIVA, 2006; TEMPRANO et al., 2006; BARCO; WONG; STENSTROM, 2008; KREBS et al., 2013).

A área impermeável encontrada foi em torno de $35 \%$, o que indica que esse valor é condizente com a impermeabilização que pode ser observada na imagem da bacia (Figura 1).

A inclinação média do conjunto de soluções foi de 9,5\%, que é próximo do valor de $10,9 \%$, e a largura da bacia, que é um parâmetro teórico sem grande representação física, ficou em torno de $750 \mathrm{~m}$. A largura e a inclinação da bacia são de suma importância, pois são valores que estão associados à velocidade de escoamento.
Os coeficientes da equação de Horton apresentaram valores próximos para a taxa de infiltração inicial $\left(f_{o}\right)$ e final $\left(f_{c}\right)$, com medianas em torno de 8,6 e $6,5 \mathrm{~mm} / \mathrm{h}$, respectivamente. O valor da taxa de infiltração inicial ficou abaixo do esperado, praticamente não havendo diferença para o encontrado na taxa final da infiltração. Isso pode indicar que para a condição do evento o solo da bacia poderia estar com um nível de umidade alto na condição do evento.

$\mathrm{Na}$ análise de sensibilidade dos parâmetros do modelo, apresentada na Figura 6, pode-se observar a variação de cada função objetivo perante a mudança de $5 \%$ no valor de cada parâmetro do modelo.

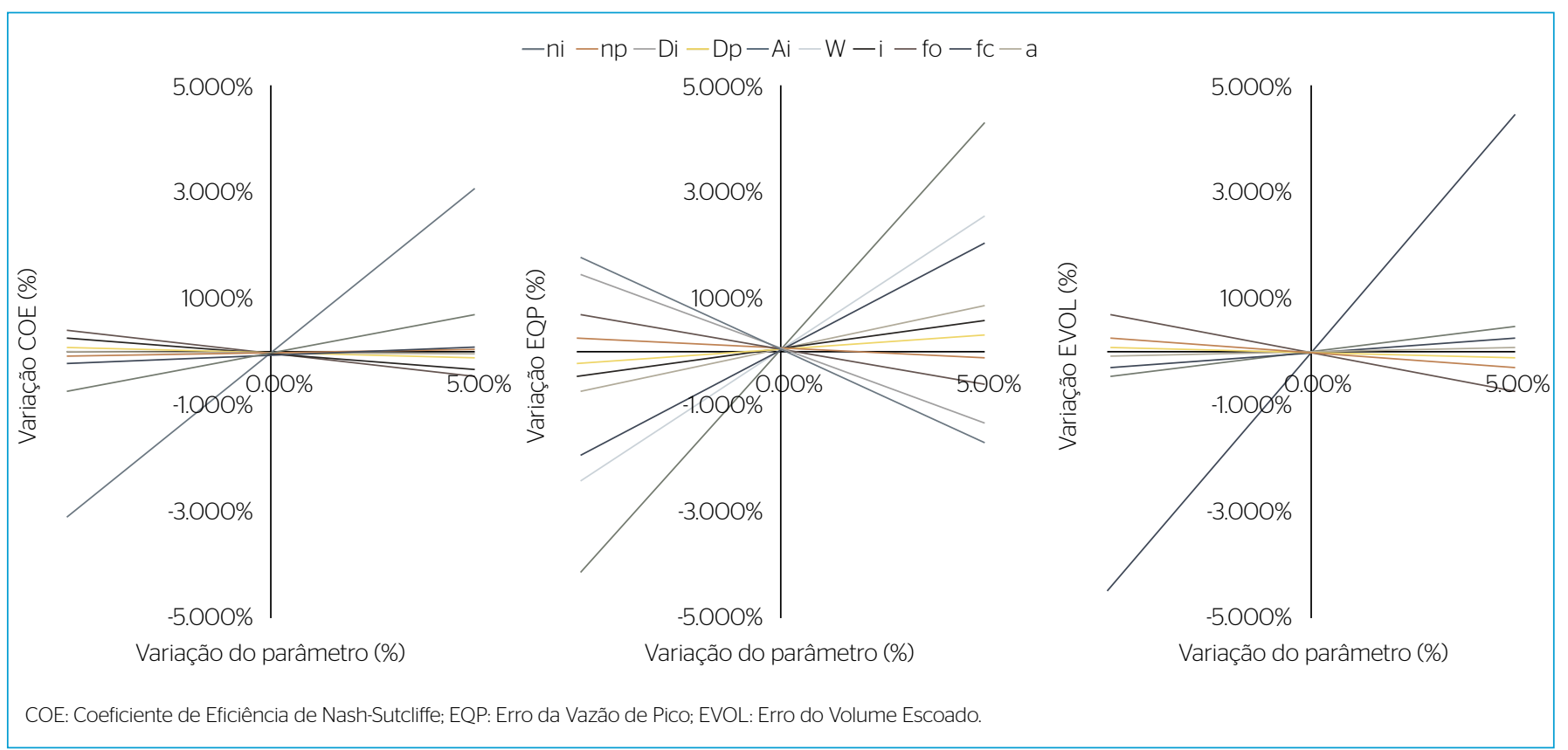

Figura 5 - Análise de sensibilidade das funções objetivo para a variação dos parâmetros.

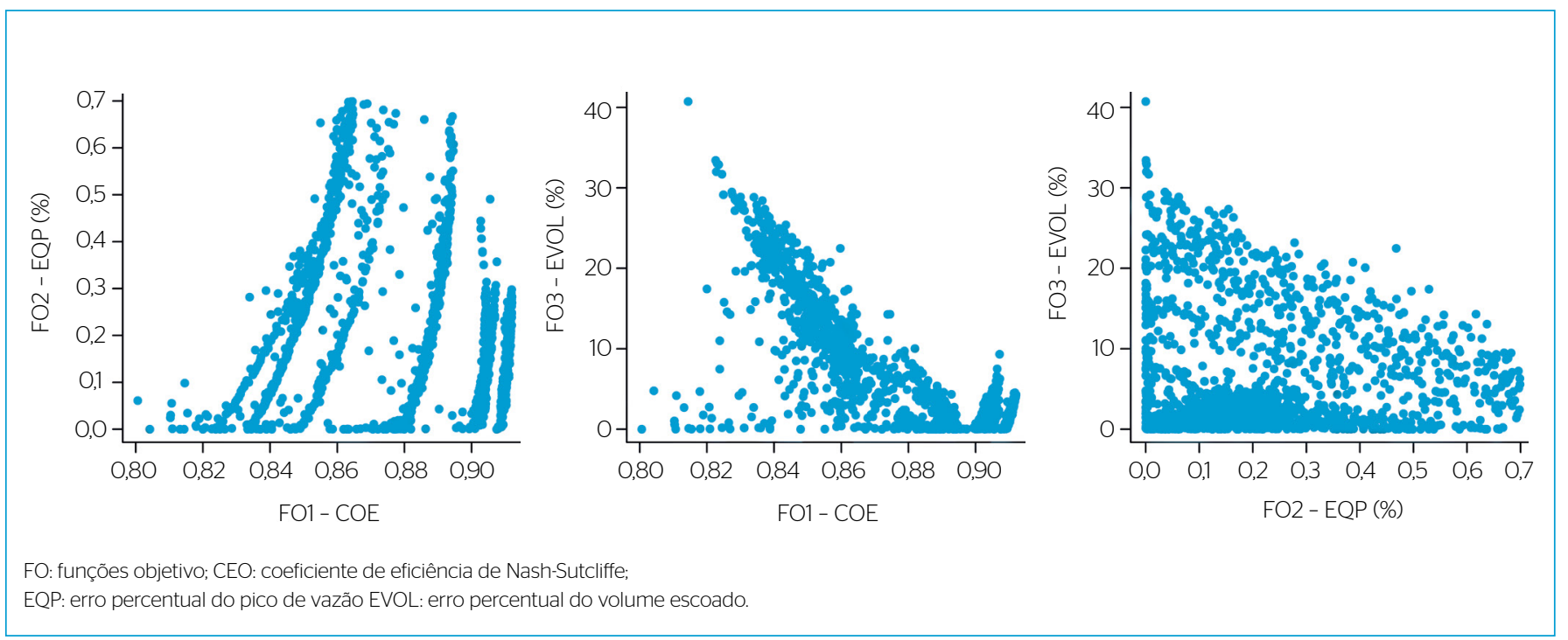

Figura 6 - Relação entre as diferentes funções objetivo para o conjunto de soluções não dominadas para o evento E1. 
A função COE foi a que apresentou menor sensibilidade, e o único parâmetro que se mostrou sensível foi $\mathrm{n}_{\mathrm{i}}$. Isso pode ser explicado em parte pela composição da função, que incorpora diversas comparações, sendo todas as vazões importantes.

Ainda de acordo com a Figura 4, para a vazão de pico, vários parâmetros demonstraram sensibilidade, principalmente o $\mathrm{W}, \mathrm{i}, \mathrm{n}_{\mathrm{i}}$ e $\mathrm{A}_{\mathrm{i}}$. Os três primeiros estão associados à velocidade da água na bacia, que interfere diretamente no tempo de concentração da bacia e consequentemente no pico. Já o último (área impermeável - $\mathrm{A}_{\mathrm{i}}$ ) está associado à quantidade de escoamento produzida, em que, para o volume gerado, foi o parâmetro que demonstrou maior sensibilidade. Esses resultados corroboram os resultados obtidos nos trabalhos de Garcia e Paiva (2006), Goldstein et al. (2010), Krebs et al. (2013) e Muleta et al. (2013).

A Figura 6 apresenta o comportamento das frentes não dominadas, onde se observa que, para as três FO, foram encontradas soluções próximas do máximo valor ótimo teórico. Logo, na distribuição de frequências (Figura 7), a maioria dos valores ficou próxima a essa mesma região, indicando uma excelente capacidade do modelo em se aproximar do ótimo individual, já que as frentes apresentam-se bem preenchidas nesta zona.

De acordo com Moriasi et al. (2007), os resultados da calibração podem ser classificados como muito bons se os valores de COE ficarem entre 0,75 e 1,0. Assim, como os valores para esse indicador foram sempre superiores a 0,8 , pode-se afirmar que, para a calibração, o comportamento do modelo foi satisfatório, uma vez que as vazões de pico e volume escoado apresentaram erros inferiores a $10 \%$, indicando um bom ajuste do modelo, devido aos valores estarem dentro da faixa de erro das medições de vazões.

Após se determinar o conjunto de soluções não dominadas, tendo sido encontradas 1.235 soluções, efetuou-se a escolha das soluções representativas desse conjunto, em que foram selecionados os melhores conjuntos com valores individuais para cada função e os que apresentaram o melhor compromisso. As melhores soluções com valores individuais para cada $\mathrm{FO}$ e as que apresentaram o melhor compromisso são apresentadas na Tabela 2 e na Figura 8.

De acordo com a Figura 8, os valores das funções apresentaram valores muito bons em todos os seis conjuntos selecionados, indicando o bom ajuste. Os valores dos parâmetros obtidos ficaram também muito próximos dos valores das características físicas da bacia, com exceção dos referentes à equação de Horton, que, no entanto, apresentou pouca sensibilidade no modelo. Isso indica que para bacias simulares o SWMM apresenta satisfatória capacidade de predição das vazões, utilizando valores dos parâmetros obtidos em campo e/ ou sensoriamento remoto.

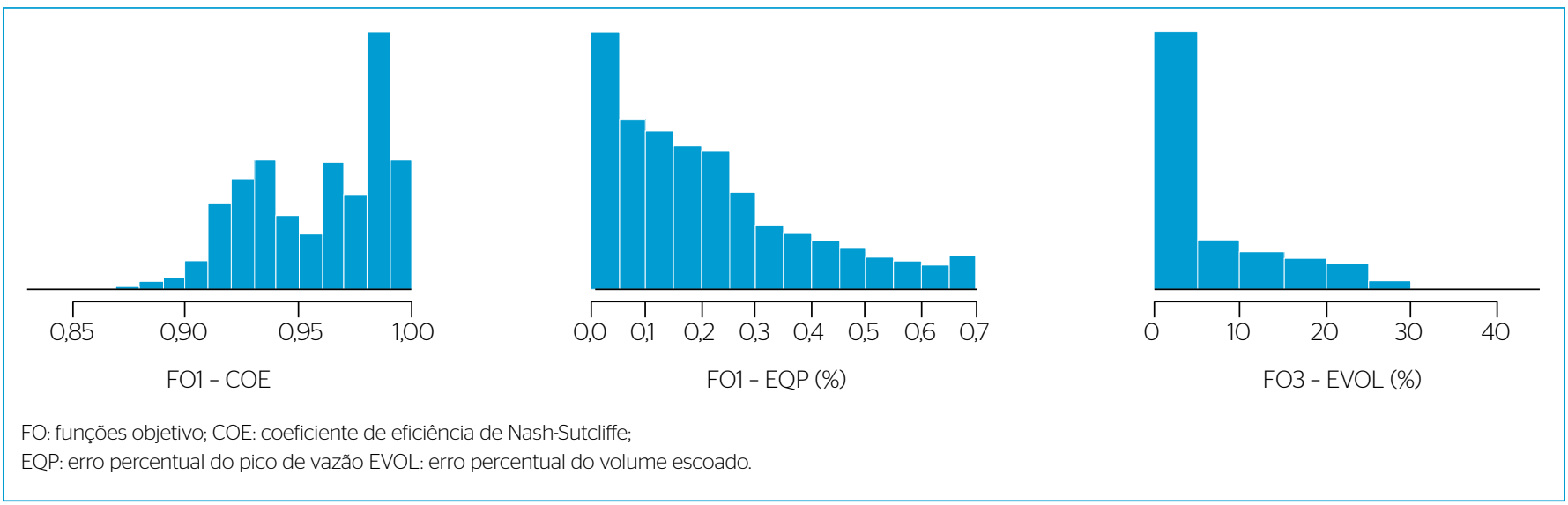

Figura 7 - Histogramas de frequência das funções objetivo para ao conjunto de soluções Pareto.

Tabela 2 - Parâmetros e valores de funções objetivo das soluções com valores mais elevados para cada funções objetivo e com o melhor compromisso.

\begin{tabular}{|c|c|c|c|c|c|c|c|c|c|c|c|c|c|}
\hline \multirow{2}{*}{ Sol. } & \multirow{2}{*}{ F01 } & \multirow{2}{*}{ FO2 } & \multirow{2}{*}{ FO3 } & \multicolumn{10}{|c|}{ Parâmetros } \\
\hline & & & & 1 & 2 & 3 & 4 & 5 & 6 & 7 & 8 & 9 & 10 \\
\hline 1 & 0,998 & 1,678 & 3,678 & 0,017 & 0,020 & 6,949 & 2,144 & 32,2 & 865,7 & 7,699 & 10,477 & 8,926 & 0,363 \\
\hline 3 & 0,979 & 1,987 & 0,000 & 0,015 & 0,065 & 5,147 & 5,953 & 33,5 & 874,4 & 6,346 & 7,961 & 4,185 & 0,415 \\
\hline 4 & 0,990 & 0,820 & 0,143 & 0,017 & 0,020 & 6,952 & 1,875 & 32,6 & 865,7 & 7,533 & 10,482 & 8,941 & 0,363 \\
\hline 6 & 0,987 & 0,631 & 0,113 & 0,017 & 0,020 & 6,952 & 1,878 & 32,6 & 865,7 & 7,685 & 10,457 & 8,946 & 0,360 \\
\hline
\end{tabular}

Sol: Soluções; FO: funções objetivo. 


\section{Validação}

Para a validação do modelo, foram utilizados os eventos E2 e E3, que têm os resultados apresentados nas Figuras 9 e 10, respectivamente. Pode-se verificar que as soluções obtidas a partir do método de melhor compromisso, soluções 4, 5 e 6, apresentaram melhor comportamento quando comparadas com aquelas de ótimos para apenas uma FO, indicando a importância de se ter mais de uma FO comandando o processo de escolha, evitando, assim, a especialização excessiva do modelo.

Para o evento E2, as soluções 1 e 3 apresentaram valores para o pico maiores que para o observado, enquanto a solução 2 mostrou

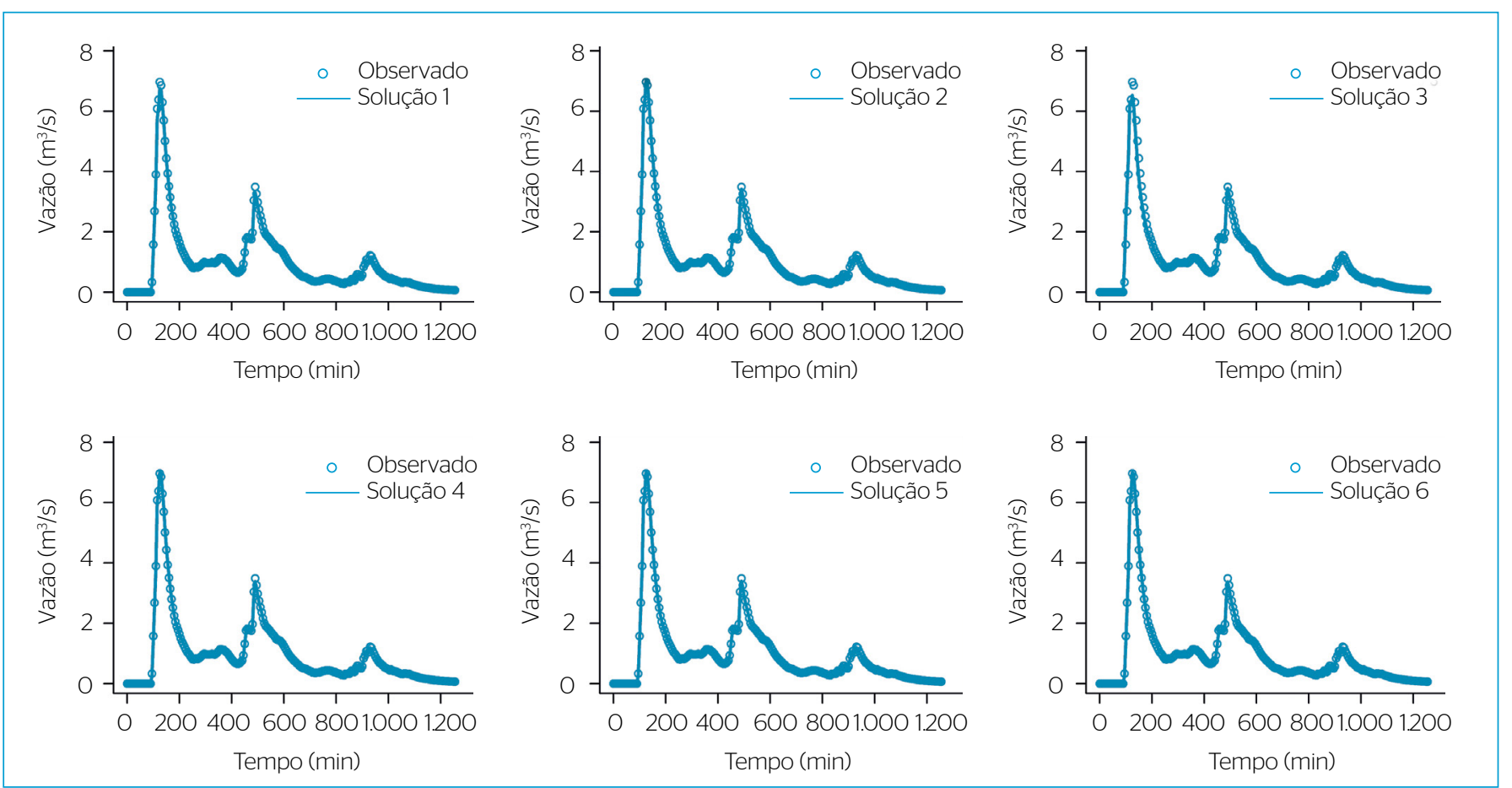

Figura 8 - Hidrogramas observados e calculados do evento E1 para diferentes conjuntos de soluções.
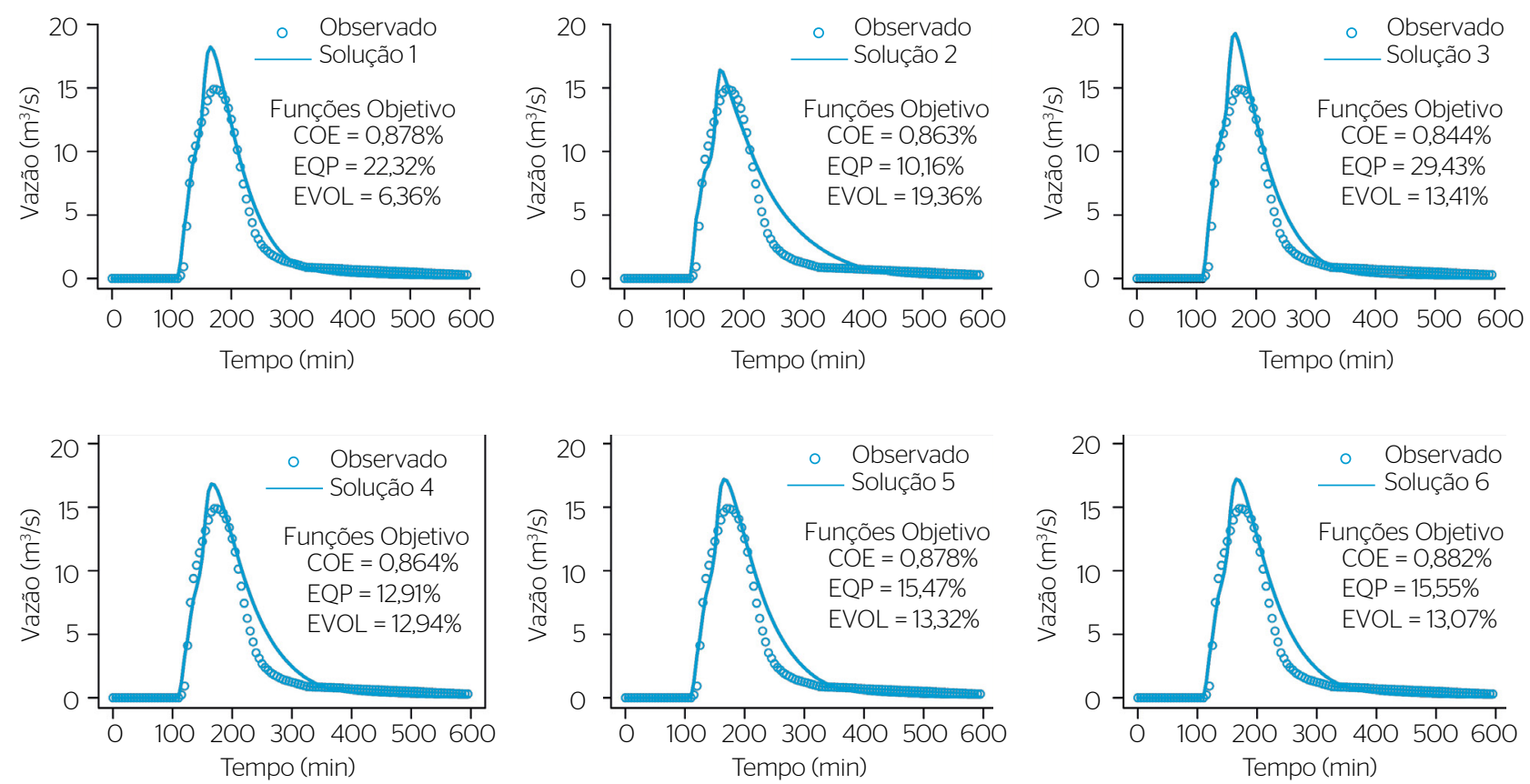

Figura 9 - Hidrogramas observados e calculados do evento E2 para diferentes conjuntos de soluções. 


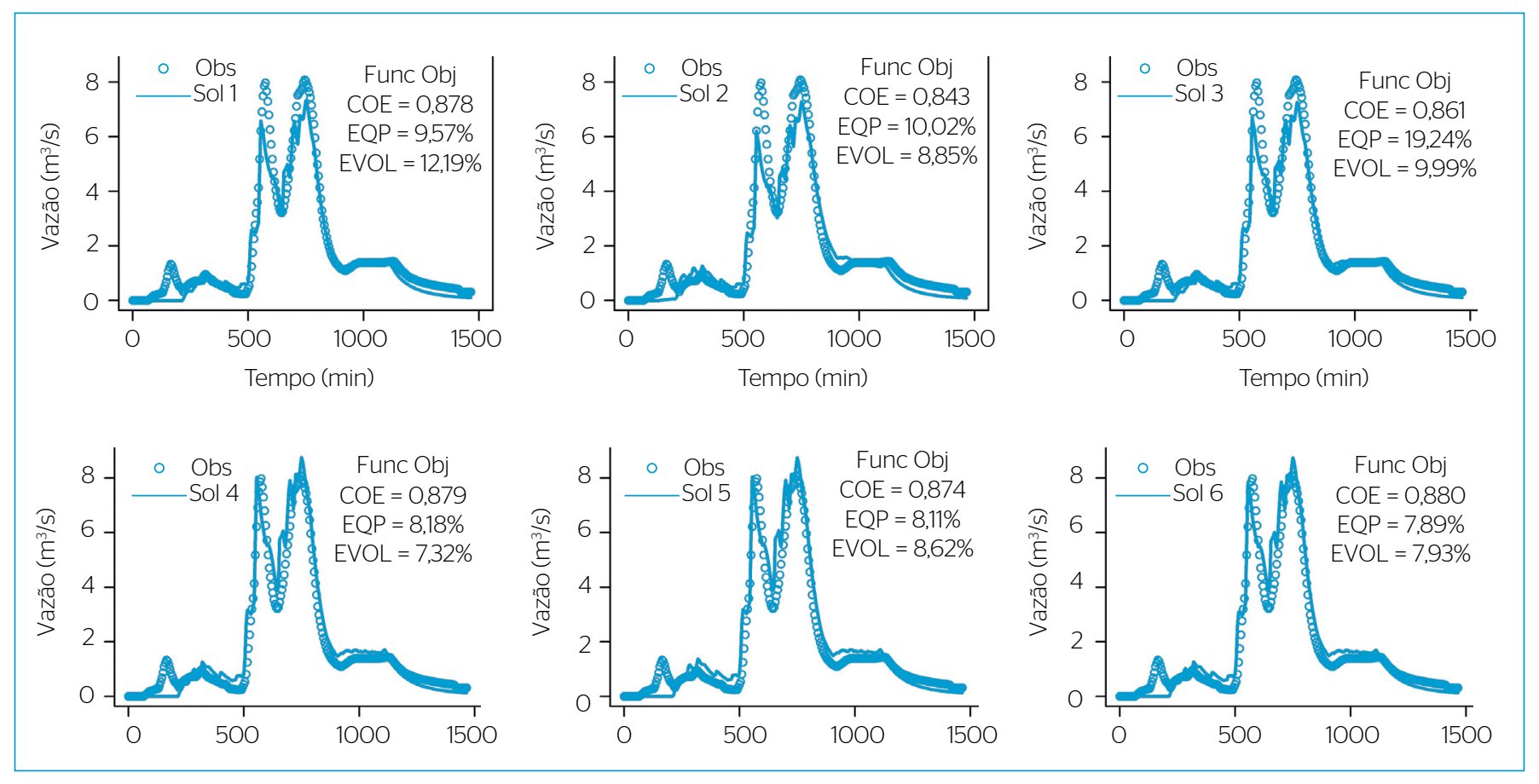

Figura 10 - Hidrogramas observados e calculados do evento E3 para diferentes conjuntos de soluções.

diferença relativamente grande em relação ao volume escoado, evidenciada pela curva de recessão acima do que o observado. As soluções de 4 a 6 apresentaram comportamentos semelhantes, com valores dos conjuntos das FO melhores que as das soluções 1, 2 e 3, o que, de certo modo, era esperado, uma vez que seus parâmetros dos conjuntos são semelhantes.

Os resultados evidenciaram a importância de se empregar métodos multiobjetivo no processo de calibração de modelos hidrológicos. Como o nível de incerteza dos modelos nestas condições é elevado e os processos de otimização são cada vez mais eficientes, o uso de apenas uma métrica para aferir a adaptação dos modelos pode causar um ajuste demasiadamente elevado para uma situação, porém não tão bom em outras condições. Por isso, as soluções baseadas em maior compatibilidade tendem a ter um melhor comportamento para uma gama maior de condições de escoamento.

\section{CONCLUSÕES}

Como os parâmetros encontrados ficaram dentro da faixa, exceção feita aos referentes ao modelo de infiltração de Horton, o modelo SWMM é passível de ser empregado, principalmente para eventos de grande intensidade, em bacias pequenas sem informações vazão, uma vez que o modelo tem uma boa representatividade física do problema.

O algoritmo de otimização apresentou satisfatória capacidade de convergência, uma vez que conseguiu atingir valores próximos aos ótimos teóricos para cada função objetivo em separado.

Os parâmetros que apresentaram maior sensibilidade foram: área impermeável, coeficiente de escoamento de Manning, largura de escoamento e inclinação da bacia.

As soluções obtidas por meio da maior compatibilidade apresentaram melhor adaptação para outras situações, indicando a importância do uso da otimização multiobjetivo em problemas de calibração de modelos hidrológicos.

\section{REFERÊNCIAS}

ASCE Task Committee. (1993) Criteria for evaluation ofwatershed models. Journal of Irrigation and Drainage Engineering, v. 119, p. 429-442.

BARCO, J.; WONG, K.M.; STENSTROM, M.K. (2008) Automatic Calibration of the U.S. EPA SWMM Model for a Large Urban
Catchment. Journal of Hydraulic Engineering. v. 134, n. 4, p. $466-474$

BARROS, L.C.; TAVARES, W.S., BARROS, I.R., RIBEIRO, P.E.A. (2O11) Integração das tecnologias sociais barraginhas e lago de múltiplo uso. Revista Brasileira de Agropecuária Sustentável, v. 1, p. 1-5. 
DAMÉ, R.C.F.; TEIXEIRA, C.F.A.; TERRA, V.S.S.; ROSSKOFF, J.L.C. (2010) Hidrograma de projeto em função da metodologia utilizada na obtenção da precipitação. Revista Brasileira de Engenharia Agrícola e Ambiental, v. 14, n. 1, p. 46-54.

DANISH HYDRAULIC INSTITUTE - DHI. (2010) Mouse: User's manual and tutorial. Horsholm (Denmark): DHI; 54 p.

DEB, K.; AGRAWAL, S.; PRATAB, A.; MEYARIVAN, T. (2000) A Fast Elitist Non-Dominated Sorting Genetic Algorithm for MultiObjective Optimization: NSGA-II. In: M. SCHOENAUER, K.; DEB, K.; RUDOLPH, G.; YAO, X.; LUTTON, E.; MERELO, J.J.; SCHWEFEL, $\mathrm{H}-\mathrm{P}$. Proceedings of the Parallel Problem Solving from Nature, VI Conference, p. 849-858.

DEB, K.; SUNDAR, J.; RAO, N.U.B.; CHAUDHURI, S. (2006) Reference Point Based Multi-objective Optimization Using Evolutionary Algorithms. International Journal of Computational Intelligence Research, v. 2, n. 3, p. 273-286.

FORMIGA, K.T.M.F. (2005) Otimização Multiobjetivo de Projetos de Redes de Distribuição de Água. Tese (Doutorado em Hidráulica e Saneamento) - Universidade de São Paulo, São Paulo.

GARCIA, J.I.B. \& PAIVA, E.M.C.D. (2006) Monitoramento Hidrológico e Modelagem da Drenagem Urbana daBacia do Arroio Cancela RS. Revista Brasileira de Recursos Hídricos, v. 11, p. 99-108.

GOLDSTEIN, A.; DIGIOVANNI, K.; MONTALTO, F. (2010) Resolution and Sensitivity Analysis of a Block-Scale Urban Drainage Model. World Environmental and Water Resources Congress 2010: p. $4720-4729$.

GUPTA, V.K. \& SOROOSHIAN, S. (1985) The automatic calibration of conceptual catchment models using derivativebased optimization algorithms. Water Resources Research, v. 21 , p. $473-485$.

KIM, B.; SANDERS, B.F.; HAN, K.; KIM, Y.; FAMIGLIETTI, J.S. (2O14) Calibration of stormwater management model using flood extent data. Proceedings of the ICE - Water Management, v. 167, p. 17-29.

KRAUSE, P.; BOYLE, D.P.; BÄSE, F. (2005) Comparison of different efficiency criteria for hydrological modelassessment. Advances in Geosciences, v. 5, p. 89-97.

KREBS, G.; KOKKONEN, T.; VALTANEN, M.; KOIVUSALO, H.; SETÄLÄ, $H$. (2013) A high resolution application of a stormwater management model (SWMM) using genetic parameter optimization. Urban Water Journal, v. 10, p. 394-410.

LIN, S. (2011) NGPM - A NSGA-/l Program in Matlab v1.4 Manual. Xian (China): Northwestern Polytechnical University. 20 p.

LIONG, S.Y; CHAN, W.T.; LUM, L.H. (1991) Knowledge-based system for SWMM runoff component calibration. Journal of Water Resources Planning Management, v. 117, p. 507-524.

LIONG, S.Y. \& IBRAHIM, Y. (1994) Estimation of peak flow and runoff volume with response surface method. Journal of Water Resources Planning Management, v. 120, n. 2, p. 161-175.
MORIASI, D.N.; ARNOLD, J.G.; VANLIEW, M.W.; BINGNER, R.L.; HARMEL, R.D.; VEITH, T.L. (2007) Model Evaluation Guidelines for Systematic Quantification of Accuracy in Watershed Simulations. Transactions of the American Society of Agricultural and Biological Engineering, v. 50, p. 885-900.

MUKOLWE, M.M.; BALDASSARRE, G.; WERNER, M.; SOLOMATINE D.P. (2014) Flood modelling: parameterisation and inflow uncertainty. Proceedings of the ICE - Water Management, v. 167, p. 51-60.

MULETA, M.; MCMILLAN, J.; AMENU, G.; BURIAN, S. (2013) Bayesian Approach for Uncertainty Analysis of an Urban Storm Water Model and Its Application to a Heavily Urbanized Watershed. Journal of Hydrologic Engineering, v. 18, p. 1360-1371.

NASH, J.E. \& SUTCLIFFE, J.V. (1970) River flow forecasting throughconceptual models: Part 1. A discussion of principles. Journal of Hydrology, v. 10, n. 3, p. 282-290.

NASCIMENTO, N.O.; MACHADO, M.L.; BAPTISTA, M.B.; SILVA, A.P. (2007) The assessment of damages caused by floods in the Brazilian context. Urban Water, v. 4, n. 3, p. 195-210.

NEUFVILLE, R.; SCHAAKE, J.; STAFFORD, J.H. (1971) Systems Analysis of Water Distribution Networks. Journal of the Sanitary Engineering Division, v. 97, n. 6, p. 825-842.

REED, P.M.; HADKA, D.; HERMAN, J.D.; KASPRZYK, J.R.; KOLLAT, J.B. (2O13) Evolutionary multiobjective optimization in water resources: The past, present, and future, Advances in Water Resources, v.51, p. 438-456.

ROSSMAN, L.A. (2010) Storm Water Management Model. version 5.0 User's Manual. Cincinnati (USA): United States Environmental Protection Agency. 295 p.

SEIBERT, J. \& MCDONNELL, J.J. (2002) On the dialog between experimentalist and modeler in catchment hydrology: use of soft information for multi-criteria model calibration. Water Resources Research, v. 38, n. 11, p. 1241-1252.

SEIBT, A.C. (2013) Modelagem hidrológica da bacia hidrográfica do Córrego Botafogo - Goiânia - GO. Dissertação (Mestrado em Engenharia do Meio Ambiente) - Universidade Federal de Goiás, Goiânia.

SRINIVAS, N.E \& DEB, K. (1994) Multiobjective Optimization Using Nondominated Sorting in Genetic Algorithms. Evolutionary Computation, v. 2, n. 3, p. 221-248.

SCHARFFENBERG, W. (2015) Hydrologic Modeling System: HEC HMS: user`s manual. Davis (USA): Hydrologic Engineering Center. 584 p.

TANG, Y.; REED, P.; WAGENER, T. (2006) How effective and efficient are multiobjective evolutionary algorithms at hydrologic model calibration? Hydrology and Earth System Sciences, v. 10, p. 289-307.

TEMPRANO, J.; ARANGO, O.; CAGIAO, J.; SUAREZ, J.; TEJERO, I. (2006) Stormwater quality calibration by SWMM: A case study in Northern Spain. Water SA, v. 32, n. 1, p. 55-63. 